\title{
UPPERCARE: A community aware environment for post-surgical musculoskeletal recovery of elderly patients
}

\author{
Conference Paper · April 2007
}

CITATIONS

0

10 authors, including:
READS

8

\section{Hugo Paredes}

Institute for Systems and Computer Engineerin..

129 PUBLICATIONS 322 CITATIONS

SEE PROFILE

Leonel Morgado

Universidade Aberta

160 PUBLICATIONS 450 CITATIONS

SEE PROFILE

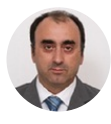

Joao Barroso

Universidade de Trás-os-Montes e Alto Douro 205 PUBLICATIONS 576 CITATIONS

SEE PROFILE

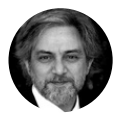

Fausto De Carvalho

Altice Labs

49 PUBLICATIONS 41 CITATIONS

SEE PROFILE

Some of the authors of this publication are also working on these related projects:

InMERSE - Exploring multimodal and immersive technologies View project 


\title{
UPPERCARE:
}

\section{A Community Aware Environment for Post-surgical Musculoskeletal Recovery of Elderly Patients}

\author{
Hugo Paredes*, João Barroso*, Leonel Morgado ${ }^{\dagger}$, Rogério Pereira $^{\ddagger}$, Ana Leal ${ }^{\S}$,

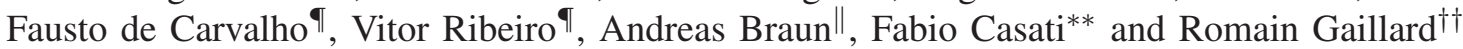 \\ *INESC TEC and Universidade de Trás-os-Montes e Alto Douro - Portugal \\ Email: \{hparedes,jbarroso\}@utad.pt \\ $\dagger$ INESC TEC and Universidade Aberta - Portugal \\ ${ }^{\ddagger}$ Espregueira-Mendes Sport Center, Saude Atlantica, S.A. - Portugal \\ ${ }^{\S}$ Dom Henrique Research Centre - Portugal \\ IAltice Labs - Portugal \\ $\|_{\text {Fraunhofer Institute for Computer Graphics Research IGD - Germany }}$ \\ ** University of Trento - Italy \\ ${ }^{\dagger \dagger}$ Centre Albert Trillat - France
}

\begin{abstract}
Disability from musculoskeletal diseases and comorbidities may lead to the worsening of social and economic well-being through a multitude of paths. Moreover since in European Union (EU) Member States it is projected that those aged 65 and over will become a much larger share (rising from $17 \%$ to $30 \%$ of the population), and those aged 80 and over (rising from $5 \%$ to $12 \%$ ) will almost become as numerous as the young population in 2060 , there is a great potential for Information and Communication Technologies (ICT) solutions for addressing the present and future living arrangements in older people.

The UPPERCARE system is meant to affect positively both the intergenerational and partners care since it contributes to decrease usability barriers and promote collaborative environments for informal and self-care. UPPERCARE is a new approach for integrated care supported by ICT systems and services, focusing on post-operative rehabilitation of musculoskeletal pathologies, having as a case study the knee post-operative scenarios of prosthetic care. This paper presents the UPPERCARE system, that provides an integrated care solution, supported ICT, for empowering self-care and adherence to rehabilitation plans through natural interfaces, gamification and cross-modal paths for community care collaboration. The system addresses current barriers from technological, clinical, social and organisational perspectives in a multidisciplinary environment. Special attention is given to the patients' needs and behaviours entailing the participation of a wide care community, including clinical and non-clinical people, associations, institutions and authorities) through an user driven interaction within the system.
\end{abstract}

\section{INTRODUCTION}

World Health Organisation (WHO) defines chronic conditions as the ones requiring "ongoing management over a period of years or decades". These conditions go beyond the conventional definition of chronic illness and cover a wide range of health problems including musculoskeletal disorders [1]. The decrease of adult mortality and fertility, and the increase in the proportion of older age groups, cause a worldwide phenomenon: the ageing population [2]. This demographic change has been transforming the societies and it is a shift in the most prevalent types of diseases, from acute infectious to chronic non- communicable diseases, such as Musculoskeletal Conditions (MSC) [3]. The MSC represent around 15-20\% of consultations in primary care and are a major cause of morbidity throughout Europe and have a substantial influence on health and quality of life inflicting an enormous cost on health and social care systems. After important surgeries, kinesiophobia, immobility and complications often result in severe and even lethal pre-existing or new co-morbidities. Moreover, as pain is the primary complaint of individuals with MSC [4] it has impacts on quality of life, independence and social participation. The high prevalence of these conditions along with population ageing is worrisome requiring appropriate policies to incorporate a strategic plan for the promotion of health and prevention of disabilities in the elderly population. Medication and physical activity control, including preventive and therapeutic exercises, are crucial to achieve positive outcomes, independence in daily activities, and to lessen the need to seek out medical care seek. Innovative strategies have been explored aiming to decrease the need of residential and admission to hospital by developing (smart) technologies that promote healthy living at home supporting older people to live as independently [5]-[7].

In preceding studies, the social factor proved to be crucial for the elderly health since the gradual deterioration of mobility becomes stronger and consequently the risk of social isolation [8], [9]. This is the context where the concept of social connectivity arises. The concept of social connectivity is multidimensional and among the most complicated in terms of definition, conceptualisation and measurement [10].

This paper presents UPPERCARE: a novel unified health platform for proactive patient centred musculoskeletal rehabilitation, through personalised multimodal and natural interfaces 
that promote collaboration between the stakeholders network and daily health sensors, providing a correlational knowledge in the care process. A paradigm breakthrough is expected in the patient-doctor relation towards a new integration perspective of proximity healthcare where each intervenient plays its role in the supply chain through an ICT compliant rehabilitation protocol. Caregivers, health entities, civil authorities, doctors, nurses, patients, and social workers are empowered to collaborate and share efforts for a care workforce in this community-centric organisational model. The proposed platform can significantly impact three of the four known major categories of intervention of musculoskeletal conditions: lifestyle, pharmacological, surgical and rehabilitative. Through the usage of innovative techniques of interaction and social integration, using 3D immersive interfaces and representations, impacts are expected in the rehabilitation of elderly patients.

As a case study, total knee arthroplasty (TKA) is introduced, as one of the most common elective operations for older people in most European countries. Physical rehabilitation is a key factor after TKA that generally begins in the hospital [11] and continues, in ambulatory, both at home and in clinics [12]. An alternative to conventional face-to-face therapy is tele-rehabilitation, encompassing active treatment and follow-up [13], rather than diagnosis and evaluation by tele-consultation. Previous studies revealed that the usage of TKA tele-rehabilitation [13] was effective, safe and easy to use, and it integrated well into current clinical. Moreover, the experience of clinical physiotherapists and participants who received treatment via showed high levels of users satisfaction [13].

\section{BACKGROUND}

Post-surgical rehabilitation after first-time total knee replacement surgery is accepted as the standard and essential treatment. Rehabilitation permits to maximize a persons functionality and independence and minimize complications such as wound infection, deep vein thrombosis, and pulmonary embolism. The patient need to recover mobility, range of motion, strength, balance and painless movement are important to prevent poor health outcomes and disability [14].

The conventional standard of rehabilitation following total joint arthroplasty is a face-to-face rehabilitation program with a physiotherapist. Evolution of the rehabilitation protocols is permanent. A standard post-surgical protocol begins one day after the surgery (or the same day as possible) with passive mobilisation of the joint. It helps to decrease risk of stiffness of the joint. Continue and motorised passive mobilisation is recommended for the knee. An other important part is the fight against oedema with massage and contention. Support on the lower limb is authorised and walk is possible immediately [14]. Home-based rehabilitation follow these same objectives. However protocols need to be adapted. Autonomisation of the patient is the main rule. Exercises can be realised alone, daily and correctly, without help of a physiotherapist.

The use of ICT in the rehabilitation process is a field that has been widely explored in recent years, following the new technological tendencies in the mobility and ubiquitous computing areas [15]. The usage of ICT for rehabilitation process is often associated with the fail to comply with the prescribed physical therapy regimes, prolonging recovery periods or accelerating decline [16]. Therefore, in most of the proposed solutions, the main goal is that the systems can improve the patients fulfilment of exercises and enhance the specialists supervision of rehabilitation tasks. Health technologies are suited to address non-compliance problems as it often results from patients explicit decision, namely: low perceived severity of symptoms; perceived ineffectiveness of the intervention; and unwillingness and inability to incorporate the treatment into everyday life. However, several authors [?] argue that digital technology can support and motivate users in health related activities. Researchers have explored social approaches to motivation, including online social network services [17] and sharing of progress to a peer group [18]. Games and entertainment were also explored as motivational tools to support exercise performance integrated into game play [19].

The Collaborative Virtual Environments (CVEs) has gained popularity for its collaboration, immersion and socialisation capabilities. These cyberspaces break with the traditional usage of ICT in a restrictive manmachine interaction promoting social interaction and the creation of close relationships. The development and growth of these virtual environments is conditioned not only by technological factors, but also by psychological and sociological factors [20]. As CVEs improve the way remote users interact with one another they are also used in the health domain, for simulation of medical procedures, training and rehabilitation [21]. The design of CVE for rehabilitation has been topic of research in the last years. Hutchinson et al. [22] propose a methodology for designing simple, flexible and adaptable technologies to be introduced into families' homes that can be applied for elderly in home rehabilitation. Axelrod et al. [23] apply Hutchinson et al. work generalising its applicability and presenting the design challenges for rehabilitation technology at home. Balaam et al. [24] illustrate some of the competing and complicated needs of participants using CVE for rehabilitation in order to assist other designers working with rehabilitation technology.

The association between CVEs and existing technologies for NUI introduce new challenges for immersion, where the body and the physical presence is carried into the virtual environment. The full body motion techniques and its suitability for shared virtual environments are crucial to the success of these environments. Normand et al. [25] present a system for Full Body Acting Rehearsal in a Networked Virtual Environment, combining immersive VR rendering techniques, network capabilities and full body tracking. According to Georgescu et al. [26], the motion capture and rendering on an avatar is one of the most promising approaches for online social interaction, know as Transformed Social Interaction TSI [27], [28]. This scenario appears as ideal for the use health technologies, as is the particular case of physical rehabilitation processes. This is enhanced by Paiva et al. [21] "the lifting of applications applied to health found [...] the great majority 
are VR-based systems". Among the various proposed systems, stands out the "Low-cost motivated rehabilitation system for post-operation exercises” presented by Brutovsky and Novak [29].

Despite the benefits of the usage of ICT in rehabilitation, Zhou et al. [30] present a study suggesting that seniors (i 65 years) have not adopted technology as part of their rehabilitation because most systems have complex set ups, are expensive, often have poor interactivity and may require large or fixed spaces which may not be available or wanted in peoples current homes. Egglestone et al. [31] conducted a study to identifying the key components for home-based stroke rehabilitation system, which were summarised in a design framework. According to the authors conclusions, the key themes and requirements for home-based rehabilitation technologies rely on the designing for wider social context; personalising system components; initiating and concluding interactions

\section{UPPERCARE}

The UPPERCARE is a novel unified health approach for proactive patient centred musculoskeletal care in TKA rehabilitation, through personalised multimodal and natural interfaces that promote collaboration between the stakeholders network and daily health sensors, providing a correlational knowledge in the care process.

\section{A. UPPERCARE TKA Rehabilitation Protocol}

Traditionally the clinical protocol associated Total Knee Arthroplasty (TKA) is divided in three phases showed in Figure 1. The development and introduction of UPPERCARE system will adjust the current procedure for the use of information and communication technologies. The protocol adaptation keeps the macro structure of the 3 phases, adjusting the procedures in each stage against the provided tools.

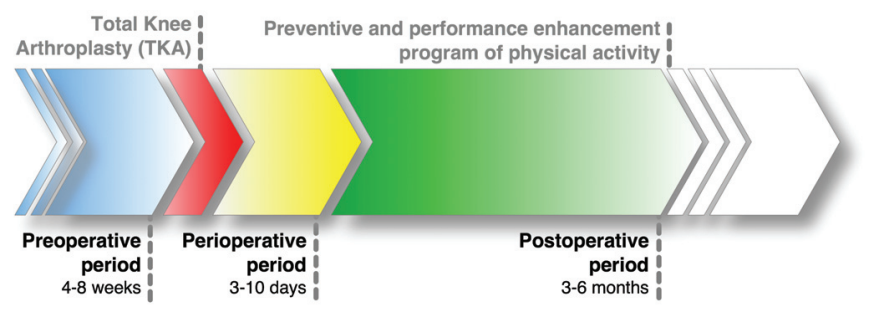

Fig. 1. TKA rehabilitation timeline

1) Preoperative period: The first period starts with a consultation with the Medical Doctor (MD) where the patient is assessed or reassessed and criteria matches indication of the patient for TKA. The screening of patients to use the UPPERCARE system follows up with an introduction to the system and its basic interfaces for the patient: UPPERCARE WebPortal and the UPPERCARE MobileApp. Using these interfaces, the patients can access to advice and information for the preparation of surgery, and a detailed description of the procedure (some of this information is now available in paperback format). The interactions of the patients with the system allow the inference of expertise in the use of information technologies (using embedded mechanisms for gathering user skills through interaction with the UPPERCARE system) that enables the adaptation of the interfaces to the user ICT profile.

2) Perioperative period: Upon surgery, the patient starts a convalescence period, with clinical supervision of around 1 week. During this period, the therapists introduce the UPPERCARE OnlineGym around the several sessions in clinical facilities. In the clinical team's meeting the direct actors in the rehabilitation process are identified, namely the clinical personal and the informal caregivers. Moreover it is defined the care flow, embedding the information flow between actors in the recovery process based on pre defined templates. Before leaving the hospital, the patient has several clinical appointments: Orthopaedic Surgeon; Physiatrist; and Physical Therapist. In the appointment with the Orthopaedic Surgeon (responsible for the surgical intervention) for medical discharge, the care flow is reviewed and validated within the patient. Moreover the Orthopaedic Surgeon also prescribes and registers in the UPPERCARE system the medication, monitoring and future consultations of the patient. The Physiatrist prescribes the recovery plan and registers in the system the patient's clinical assessments. In the final appointment, with the Physical Therapist, the recovery plan implementation is defined, including the recovery, preventive and contraindicated exercises. Furthermore, the clinician also registers the plan in the UPPERCARE system and demonstrates it to the patient in UPPERCARE OnlineGym, with face-to-face supervision of the therapist.

3) Postoperative period: Following the perioperative period the patient returns home and starts the rehabilitation plan in the post-operative period. The UPPERCARE system is part of the everyday life of the patient, through the monitoring, exercising, caregiving and assistance provided. During the first two weeks the chances of complications are high with risk of infection. Therefore, there is a strict monitoring of temperature, pain, local warmness, wound healing and vital signs to ensure that the caregivers and the clinicians in the rehabilitation centre follow the patient. Thus, gathering this information helps to address a suspected periprosthetic infections by referring the patient though a diagnostic process for early detection and treatment. Informal monitoring is performed by wearable devices that trigger alarms to the patient and caregivers in case suspect changes in the defined thresholds. The action required is the validation of the results within medical equipment connected to the UPPERCARE system. Beyond this particular situation, the patient should verify their vital signs twice a day, according to a schedule defined. If not, a notification is triggered to the caregiver following the care flow defined. The caregiver should notify the system that will proceed with the verification of the situation. Else, after a defined time threshold for the situation, the following caregiver is contacted. After a successful notification of care, the system will provide the caregiver the procedure to follow. This operation can 
be supervised by clinical personal at the UPPERCARE call centre. If the situation remains, and according to the defined care flow, a response to the situation is provided. The same procedure is followed when the user do not take his medication or do not perform the physiotherapy rehabilitation programme which includes therapeutic exercise (strengthening and range of motion exercises), transfer and gait training and go through simple but very important instructions/counselling related to activities of daily living, previewed in the rehabilitation plan.

\section{System ARchitectURE}

The UPPERCARE system provides an accessible system with interfaces adapted to each user, their capabilities and needs, following current and future standards. Boosted by an ecosystem for promoting collaboration and interaction between the stakeholders in the rehabilitation process for its full compliance, the system architecture reflects the protocol needs. As a result from the merge of requirements stand out in a modular architecture with high message flow and information registration throughput, which enables rapid integration of new components and the development and maintenance of the system by future needs or applications in other case studies (Total Hip Arthroplasty THA, Osteoporosis, back pain, rheumatoid arthritis RA, etc.)

\section{A. UPPERCARE Ecosystem}

The UPPERCARE ecosystem defines three rehabilitation environments: personal, clinical and community. In this ecosystem act the key players: patients, clinicians (medical doctors, therapists, nurses) and caregivers (informal caregivers, family, friends).

These privileged UPPERCARE usage environments is directly derived from the analysis of the rehabilitation protocol. They aim to highlight the interactions between human actors, having regard such that is the main objective of the system. The UPPERCARE stands out from ambient assited living systems and e-health for their their collaboration and socialisation features.

In the clinical environment the clinical-patient relation is individualised and specific to each patient. Typically this scenario is associated to medical appointments and exemplification of processes using the UPPERCARE system in the hospital (in the perioperative period). Back home, the self-care is privileged. The interactions are usually in the virtual gym (UPPERCARE OnlineGym - Figure 2), where therapists and multiple patients share the same (virtual) space and interact. In a broader environment, the community is also included on the interactions, where informal caregivers (and all other member of the care chain of the patient) play a key role in the compliance of the rehabilitation plan.

\section{B. Physical Architecture}

The physical architecture of UPPERCARE system follows a client-server architectural style. To ensure the highest standards of availability and reliability of the server side is physically hosted in the Cloud. Given the logical architecture

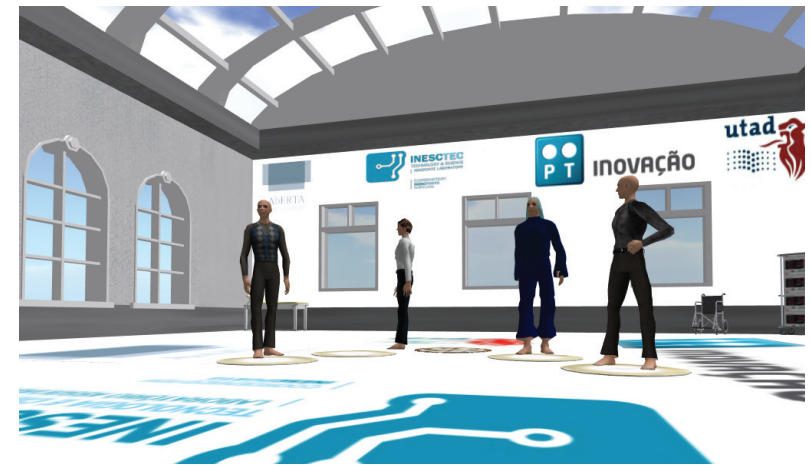

Fig. 2. UPPERCARE OnlineGym - OnlineGym project [32]

requirements, which will be further described, a Platform as a Service (PaaS) strategy is used, decoupling the platform management and infrastructure from the development, maintenance and evolution of the system. To ensure legislation requirements of clinical data storage, the system data and clinical records can be stored in a private cloud our in-house hosting service. This physical modularity ensures a global spread of the platform meeting the legal obligations of each country.

The client side of the physical architecture includes several components as it requires multimodal interaction and adaptation to the contexts provided for the UPPERCARE ecosystem. The core component is the UPPERCARE rehabilitation kit, which is given to the patient when he/she returns home for the postoperative period. This kit is a set of hardware that is used by the patient as part of their rehabilitation process, including wearable sensors for monitoring biological parameter and biomechanical settings using consumer electronics devices (wearable activity tracker). The homeostatic deviations of the values obtained by these sensors are indicative and represent a first alarm, which must be evidenced by the measuring of medical devices included in the "UPPERCARE rehabilitation kit" with the in-house sensors. The measurements of vital parameters with these devices are part of the rehabilitation plan and are scheduled in patient daily routine. The intake the medication, which is dispensed automatically and controlled by the system, follows the same principle.

\section{Logical Architecture}

UPPERCARE adopts a modular approach to ensure the separation of concerns, enhancing the evolution, adaptation and maintenance of the platform in a strong scientific and technological foundation. The platform integrates current tools and technologies as key building blocks. The model follows the traditional structure for enterprise application integration with a enterprise bus to which the modules are connected (Figure 3). Given the requirements for the exchange of messages between the different components it is required that the system provide an interconnecting backbone to ensure the high throughput of information exchange for pervasive patient monitoring. 


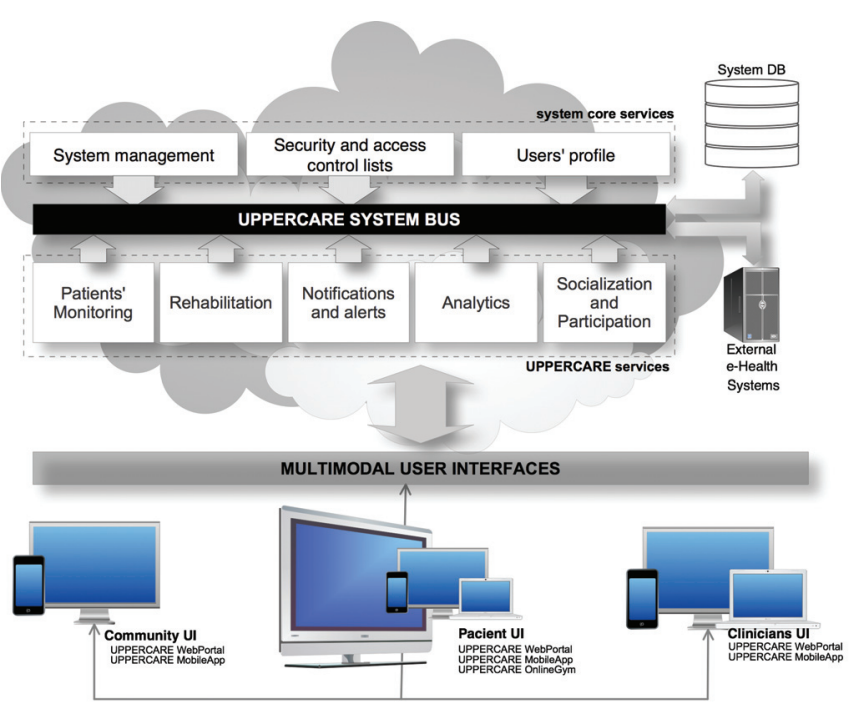

Fig. 3. UPPERCARE architecture

Three transversal modules for core functions support the system:

Users profile: Manages the users' profiles, including patients, clinicians and caregivers. In the patient specific case, this module is also responsible for managing the care flow, and relating it to the registered caregivers in the system as well as the clinical history.

Security and access control lists (ACL): Manages the global permissions and access to information, defining, implementing, monitoring and auditing the defined security policies. In a system whose core function is the interaction and collaboration between the various actors in the rehabilitation process becomes imperative to centrally and transparently manage all access and information flow.

System management: Orchestrates the integration and defines the procedures and maintenance, providing a dashboard for the administrator. This module also ensures the flows definitions and business processes as well as their monitoring and adaptation. Traditional system administration functions are also ensured by this component, such as user and components management and system monitoring.

To support UPPERCARE specific requirements for ICT based rehabilitation protocol five modules are defined:

Participation and socialisation module: Defines procedures and tools that enable the implementation of socialisation dynamics. This strategy is associated with the creation of small communities of patients sharing the OnlineGym and exchanging experiences. Furthermore implements gamification strategies for the performing exercises and fulfilment of rehabilitation objectives. The module is also responsible for providing traditional collaboration tools for system's actors collaboration, such as chat, private messaging, files exchange/sharing and audio/videoconference.

Rehabilitation module: Integrates the mechanisms for enabling the patient's rehabilitation, including the scheduling of exercises and medication.

Patients monitoring module: Provides integrated services for monitoring physiologic and biomechanical signals obtained by sensors (wearable and in-house) integrated into UPPERCARE system. The module is responsible for the standardisation of data, parameterisation, anonymization, storage, and visualisation. It relates to the physical rehabilitation module for obtaining

Notifications and alerts module: Raising events following the rehabilitation care flow defined for each patient. This component defines three types of notification: informative; regular; and priority. Each is linked to a new cycle of trigger events, as it can is necessary to follow up with notifications if no action taken by the notified user.

Analytics module: Provide a set of tools to clinicians for correlating information regarding the evolution of a patient and to define metrics with associated warnings. The module can be seen as a setting for sensors composition with combinatorial logic for processing and visualising the results. Besides this function, the module has sensors for the extraction of monitored parameters, such as analysis of body movement in exercises to extract, among others, the angular speed.

The UPPERCARE system is seen by its users through multimodal interfaces, which can be used in various contexts, as provided by the defined ecosystem. For patients, the interfaces are adapted by the skills assessed by the system. This adaptation aims to restrict the number of options available to the user. The UPPERCARE WebPortal allows the access to system functionality for patients, clinicians and caregivers. Through this interface they can access the information provided in each phase of the rehabilitation process. It is complemented by UPPERCARE Mobile App that is used in smartphones and allows notification anywhere / anytime. In addition, the Mobile App is also possible to access to the rehabilitation process information, in particular the recent history and the next stages. UPPERCARE OnlineGym is the core interface for the patient, allowing to accomplish the rehabilitation exercises at home and interaction with the other patients in the immersive virtual environment.

\section{Final REMARKS}

The major challenges of UPPERCARE are (1) enhancing the participative adherence to TKA rehabilitation plan through social connectivity and gamification strategies; (2) monitor physiologic and biomechanical patient parameters and provide an integrated and orchestrated response, involving family, friends, caregivers and clinicians in the rehabilitation process; (3) provide an integrated environment, accessible through natural user interfaces for fulfilment of the rehabilitation plan, including, physical exercises, accurate monitoring and medication; and (4) randomised clinical trials to prove the proposed TKA rehabilitation protocol.

As a result of the implementation of UPPERCARE, it is expected a reduction on admissions days spent in care institutions due to enhancement of interventions such us monitoring and treatment deliver regimens, pharmacological or rehabilitative, 
directly related with postoperative condition or patient comorbidities. This allows prompt access and adherence to treatment, and wider. The ICT platform, with its proactive features, enables to respond to rehabilitation requirements, prescribed treatment for the health problem in question and associated comorbidities, and also to homeostatic deviations, as suggested in very recent evidence-based reports. The expected reduction in complications and long-term disability from orthopaedic surgical interventions and/or chronic diseases such as rheumatism and particularly arthritis, often reported as leading causes of disability among elderly people, will add significant health, social and economic benefits.

The evaluation of these impacts will be carried out with the implementation of randomised clinical trials (RCT) in a prospective study including experimental and control groups of users selected from eligible patients based on a set of predictive factors and inclusion criterias.

\section{ACKNOWLEDGMENT}

This work was supported by Project "NanoSTIMA: Macroto-Nano Human Sensing: Towards Integrated Multimodal Health Monitoring and Analytics/NORTE-01-0145-FEDER000016" financed by the North Portugal Regional Operational Programme (NORTE 2020), under the PORTUGAL 2020 Partnership Agreement, and through the European Regional Development Fund (ERDF).

\section{REFERENCES}

[1] W. H. Organization et al., "World report on disability," 2011.

[2] J. Bongaarts, "United nations department of economic and social affairs, population division world mortality report 2005," Population and Development Review, vol. 32, no. 3, pp. 594-596, 2006.

[3] V. S. Miranda, B. Vivielle, L. A. Machado, and J. M. D. Dias, "Prevalence of chronic musculoskeletal disorders in elderly brazilians: a systematic review of the literature," BMC musculoskeletal disorders, vol. 13, no. 1, p. 1, 2012.

[4] M. S. G. Dellaroza, C. A. d. M. Pimenta, and T. Matsuo, "Prevalence and characterization of chronic pain among the elderly living in the community," Cadernos de Saúde Pública, vol. 23, no. 5, pp. 1151-1160, 2007.

[5] A. J. Pearce, B. Adair, K. Miller, E. Ozanne, C. Said, N. Santamaria, and M. E. Morris, "Robotics to enable older adults to remain living at home," Journal of aging research, vol. 2012, 2012.

[6] M. E. Morris, B. Adair, K. Miller, E. Ozanne, R. Hansen, A. J. Pearce, N. Santamaria, L. Viega, M. Long, and C. M. Said, "Smart-home technologies to assist older people to live well at home," Journal of aging science, vol. 1, no. 1, pp. 1-9, 2013.

[7] G. Demiris, B. K. Hensel et al., "Technologies for an aging society: a systematic review of smart home applications," Yearb Med Inform, vol. 3, pp. 33-40, 2008.

[8] B. N. Uchino, "Understanding the links between social ties and health on building stronger bridges with relationship science," Journal of Social and Personal Relationships, vol. 30, no. 2, pp. 155-162, 2013.

[9] J. Holt-Lunstad, T. B. Smith, and J. B. Layton, "Social relationships and mortality risk: a meta-analytic review," PLoS Med, vol. 7, no. 7, p. e1000316, 2010.

[10] B. Pescosolido, "Social connectedness in health, morbidity and mortality, and health carethe contributions, limits and further potential of health and retirement study," in Forum Health Econ Policy, vol. 14, no. 3, 2011, p. 11.

[11] R. W. Bohannon and J. Cooper, "Total knee arthroplasty: evaluation of an acute care rehabilitation program." Archives of physical medicine and rehabilitation, vol. 74, no. 10, pp. 1091-1094, 1993.

[12] E. A. Rankin, G. S. Alarcòn, R. W. Chang, L. M. Cooney Jr et al., "Nih consensus statement on total knee replacement december 8-10, 2003," Journal of Bone and Joint Surgery, vol. 86, no. 6, p. 1328, 2004.
[13] P. G. Forducey, W. D. Ruwe, S. J. Dawson, C. Scheideman-Miller, N. B. McDonald, and M. R. Hantla, "Using telerehabilitation to promote tbi recovery and transfer of knowledge," NeuroRehabilitation, vol. 18, no. 2, pp. 103-111, 2003.

[14] K. J. Bozic, S. M. Kurtz, E. Lau, K. Ong, V. Chiu, T. P. Vail, H. E. Rubash, and D. J. Berry, "The epidemiology of revision total knee arthroplasty in the united states," Clinical Orthopaedics and Related Research $囚$, vol. 468, no. 1, pp. 45-51, 2010.

[15] I. Raso, R. Hervás, and J. Bravo, "m-physio: personalized accelerometerbased physical rehabilitation platform," Proceedings of UBICOMM, vol. 10 , pp. 416-421, 2010.

[16] H. Chandra, I. Oakley, and H. Silva, "Designing to support prescribed home exercises: understanding the needs of physiotherapy patients," in Proceedings of the 7th Nordic Conference on Human-Computer Interaction: Making Sense Through Design. ACM, 2012, pp. 607-616.

[17] S. A. Munson, D. Lauterbach, M. W. Newman, and P. Resnick, "Happier together: integrating a wellness application into a social network site," in International Conference on Persuasive Technology. Springer, 2010, pp. 27-39.

[18] J. J. Lin, L. Mamykina, S. Lindtner, G. Delajoux, and H. B. Strub, "Fishnsteps: Encouraging physical activity with an interactive computer game," in International Conference on Ubiquitous Computing. Springer, 2006, pp. 261-278.

[19] J. Yim and T. Graham, "Using games to increase exercise motivation," in Proceedings of the 2007 conference on Future Play. ACM, 2007, pp. 166-173.

[20] H. Paredes and F. M. Martins, "Social interaction regulation in virtual web environments using the social theatres model," Journal of Network and Computer Applications, vol. 35, no. 1, pp. 3-19, 2012.

[21] P. V. de Farias Paiva, L. S. Machado, and A. M. G. Valença, "A virtual environment for training and assessment of surgical teams," in Virtual and Augmented Reality (SVR), 2013 XV Symposium on. IEEE, 2013, pp. 17-26.

[22] H. Hutchinson, W. Mackay, B. Westerlund, B. B. Bederson, A. Druin, C. Plaisant, M. Beaudouin-Lafon, S. Conversy, H. Evans, H. Hansen et al., "Technology probes: inspiring design for and with families," in Proceedings of the SIGCHI conference on Human factors in computing systems. ACM, 2003, pp. 17-24.

[23] L. Axelrod, G. Fitzpatrick, J. Burridge, S. Mawson, P. Smith, T. Rodden, and I. Ricketts, "The reality of homes fit for heroes: design challenges for rehabilitation technology at home," Journal of Assistive Technologies, vol. 3, no. 2, pp. 35-43, 2009.

[24] M. Balaam, S. Rennick Egglestone, A.-M. Hughes, T. Nind, A. Wilkinson, E. Harris, L. Axelrod, and G. Fitzpatrick, "Rehabilitation centred design," in CHI'10 Extended Abstracts on Human Factors in Computing Systems. ACM, 2010, pp. 4583-4586.

[25] J.-M. Normand, B. Spanlang, F. Tecchia, M. Carrozzino, D. Swapp, and M. Slater, "Full body acting rehearsal in a networked virtual environmenta case study," Presence: Teleoperators and Virtual Environments, vol. 21, no. 2, pp. 229-243, 2012.

[26] A. L. Georgescu, B. Kuzmanovic, D. Roth, G. Bente, and K. Vogeley, "The use of virtual characters to assess and train non-verbal communication in high-functioning autism," Frontiers in human neuroscience, vol. 8, p. 807, 2014.

[27] J. Blascovich and J. Bailenson, Infinite reality: Avatars, eternal life, new worlds, and the dawn of the virtual revolution. William Morrow \& Co, 2011.

[28] N. C. Krämer, "Nonverbal communication," Human behavior in military contexts, pp. 150-188, 2008.

[29] J. Brutovsky and D. Novak, "Low-cost motivated rehabilitation system for post-operation exercises," IEEE Eng Med and Biol Soc, New York, pp. 6663-6666, 2006

[30] H. Zhou and H. Hu, "Human motion tracking for rehabilitationa survey," Biomedical Signal Processing and Control, vol. 3, no. 1, pp. 1-18, 2008.

[31] S. R. Egglestone, L. Axelrod, T. Nind, R. Turk, A. Wilkinson, J. Burridge, G. Fitzpatrick, S. Mawson, Z. Robertson, A. M. Hughes et al., "A design framework for a home-based stroke rehabilitation system: Identifying the key components," in $20093 r$ International Conference on Pervasive Computing Technologies for Healthcare. IEEE, 2009, pp. $1-8$.

[32] F. Cassola, L. Morgado, F. de Carvalho, H. Paredes, B. Fonseca, and P. Martins, "Online-gym: a $3 \mathrm{~d}$ virtual gymnasium using kinect interaction," Procedia Technology, vol. 13, pp. 130-138, 2014. 